\title{
Interview with Greta Panova
}

\section{Toufik Mansour}

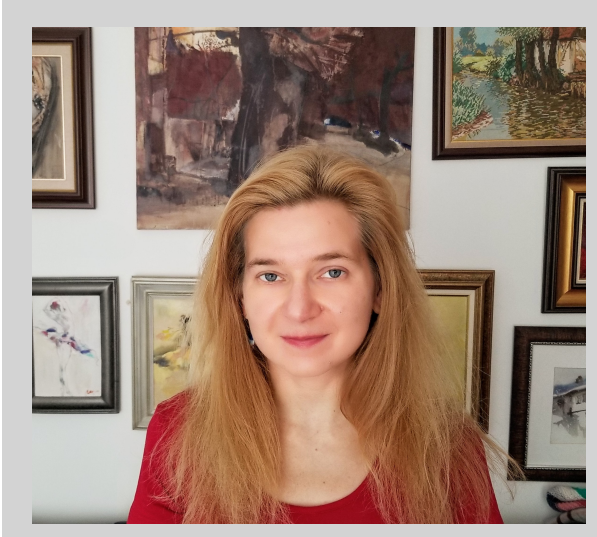

Greta Panova completed her undergraduate studies at the Massachusetts Institute of Technology, where she obtained two bachelor's degrees: in Mathematics and in Electrical Science and Engineering. She completed her master's studies at the University of California, Berkeley. She obtained a Ph.D. from Harvard University in 2011, under the direction of Richard Stanley. She currently holds the position of Associate Professor (with tenure) at the University of Southern California. From 2011 up to 2014 she was a Simons Postdoctoral Fellow at the University of California Los Angeles. During the period 2014-2018, she was an Assistant Professor, promoted to Associate (with tenure), at the University of Pennsylvania. During the period 2017-2018, she was a Von Neumann Fellow at the Institute for Advanced Studies in Princeton. Professor Panova has given numerous talks at many conferences and seminars. We list here some of her plenary talks: Triangle Lectures in Combinatorics, Greensboro, NC. (2016); Formal Power Series and Algebraic Combinatorics, London, UK (2017); Algebraic and Enumerative Combinatorics Conference, Okayama, Japan (2018). Greta Panova received many prizes and awards with the most recent one, the IMI prize for 2020, the prize awarded by the Institute of Mathematics and Informatics of the Bulgarian Academy of Sciences. She is currently one of the Editors-in-chief of The Electronic Journal of Combinatorics.

Mansour: Professor Panova, first of all, we would like to thank you for accepting this interview. Would you tell us broadly what combinatorics is?

Panova: Thank you for inviting me, it is an honor to be interviewed.

Combinatorics is generally speaking the area of mathematics that studies discrete objects without too much underlying structure and relations, which is what distinguishes it from algebra and number theory. Combinatorics itself has many subareas which do not necessarily interact much with each other graph theory, enumerative, analytic, algebraic combinatorics can all be very different and even within themselves divided. Combinatorics has both its methods and its problems, the methods could be applied to other fields, and the problems solved with external tools, all of which make this area very diverse, wellconnected to others, and its boundaries hard to define.

Mansour: What do you think about the development of the relations between combinatorics and the rest of mathematics?

Panova: Combinatorics is centrally positioned within mathematics. Combinatorial objects, in their simple structure, could manifest in external problems and then combinatorial methods become powerful tools. For example, at the heart of algebraic combinatorics lie enumerative techniques and symmetric functions, which directly apply in representation theory to understand module dimensions and structure through multiplicities. We can see such connections everywhere - in probability

The authors: Released under the CC BY-ND license (International 4.0), Published: April 2, 2021

Toufik Mansour is a professor of mathematics at the University of Haifa, Israel. His email address is tmansour@univ.haifa.ac.il 
and statistical mechanics, algebra and number theory, algebraic geometry, topology... Combinatorics goes beyond math, as it is among the main tools in theoretical computer science, game theory, etc. Moreover, the interaction is two-way, as algebraic and probabilistic methods play an important role and seemingly simple combinatorial problems have been solved with the powerful machinery of other fields. For example, algebraic geometry has been used to prove the unimodality of combinatorial sequences as in the works of Richard Stanley ${ }^{1}$ in the 80s and June $\mathrm{Huh}^{2}$ much more recently; Haiman's ${ }^{3}$ proof of the $n$ ! conjecture is another example.

Mansour: What have been some of the main goals of your research?

Panova: I try to find connections with other fields, expand the application of combinatorial methods there and also solve combinatorial problems using external methods. One direction is the study of interacting particle systems, dimer models in statistical mechanics which often benefit from the use of symmetric functions; and in the opposite direction probabilistic methods can explain the asymptotics of combinatorial quantities. Another direction is computational complexity in theoretical computer science, where a good understanding of structure constants in algebraic combinatorics (e.g. the Kronecker and plethysm coefficients) could lead to computational lower bounds. This is the basis of geometric complexity theory (GCT) which aims to distinguish the algebraic version of $\mathrm{P}$ vs $\mathrm{NP}$, the $\mathrm{VP}$ vs $\mathrm{VNP}$ problem, by exploiting the symmetries of the universal polynomials - the permanent and determinant. Unfortunately, our work on Kronecker and plethysm coefficients and disprove of the weak GCT conjecture revealed that the stepping stones in GCT are higher than we can currently reach ${ }^{4,5}$. The connections between algebraic combinatorics and computational complexity extend further beyond these applications, and another goal is to understand combinatorial quantities through the prism of complexity classes and explain formally why we do not have "nice" formulas, or even "combinatorial interpretations" sometimes.

Mansour: We would like to ask you about your formative years. What were your early experiences with mathematics? Did that happen under the influence of your family or some other people?

Panova: My parents are both very far from mathematics, although as a law professor (my mother) and as a medical doctor (my father), education in my family has always been highly valued. My mother was steering me to study languages early on, which I greatly resisted, and instead spent my time at home quietly drawing and disassembling things out of $\mathrm{cu}^{-}$ riosity and desire to build something else out of their parts resulting in inevitable destruction and sometimes injury. My memories from elementary school are quite blank in the cloud of my peer's hostility. When I was in 4th grade my mother won a Humboldt fellowship and we spent one year in Germany, where I started exhibiting some math skills recognized by the teachers. Back in Bulgaria, I went to a better middle school, and after 7th grade I was admitted to and enrolled in the National HighSchool of Mathematics and Sciences, defeating my mother's push to go to a language highschool by failing the literature entrance exam. In high school, I started going to math competitions, which turned out to be actually fun. I ultimately made it to the national team for the IMO, where I got gold and two silver medals. These were my entrance ticket to MIT and the rest is in my CV. I must add though, that despite all the math I was doing in high school, during my entire childhood I wanted to become an architect and that is what I started with at MIT.

Mansour: Were there specific problems that made you first interested in combinatorics?

Panova: No specific problem, but rather the

\footnotetext{
${ }^{1}$ R. P. Stanley, Log-concave and unimodal sequences in algebra, combinatorics, and geometry, In Graph theory and its applications: East and West (Jinan, 1986), volume 576 of Ann. New York Acad. Sci., pages 500-535. New York Acad. Sci., New York, 1989.

${ }^{2} \mathrm{~J}$. Huh, Milnor numbers of projective hypersurfaces and the chromatic polynomial of graphs, J. Amer. Math. Soc. 25:3 (2012), 907-927.

${ }^{3}$ M. Haiman, Hilberts schemes, polygraphs, and the Macdonald positivity conjecture, J. Amer. Math. Soc. 14:4 (2001), 941-1006. ${ }^{4}$ C. Ikenmeyer and G. Panova, Rectangular Kronecker coefficients and plethysms in geometric complexity theory, Adv. Math. $319,(2017), 40-66$.

${ }^{5}$ P. Bürgisser, C. Ikenmeyer, and G. Panova, No occurrence obstructions in geometric complexity theory, J. Amer. Math. Soc. 32 (2019), 163-193.
} 
problem style as a whole. I liked its problemsolving nature and the fact that I could start working on a problem without much background knowledge.

Mansour: What was the reason you chose Harvard University for your Ph.D. and your advisor Richard Stanley?

Panova: Initially my intentions were to do algebraic number theory and went to Harvard as one of the best places for that. After gaining more exposure across math fields, I carefully reexamined my interests and what I liked about math and decided to do combinatorics. I knew Richard Stanley from the freshman problem-solving seminar at MIT and it all seemed like a natural choice.

Mansour: What was the problem you worked on in your thesis?

Panova: There were several little problems I solved during my Ph.D. and some went into my thesis $6,7,8$. They were all some form of enumeration of permutations or tableaux using tools from algebraic combinatorics like symmetric functions, RSK, etc. The main problem was the enumeration of standard Young tableaux of certain truncated shapes, the ones obtained by removing a partition from the upper right corner of a Young diagram (in English notation). Such objects had not been studied before. Ron Adin and Yuval Roichman had noticed experimentally that for certain shapes the number of SYTs (linear extensions) seems to have product formulas since they did not have large prime factors ${ }^{9}$. They had asked Richard Stanley about this phenomenon and he passed it on to me to solve. In order to solve it, I used symmetric functions, complex analysis, volumes of polytopes, etc, and figuring all these techniques out was quite enlightening to me and got me interested in their natural continuation within integrable probability ${ }^{10}$.
Mansour: What would guide you in your research? A general theoretical question or a specific problem?

Panova: Usually both. I had to start with some area I want to understand or find connections to and then look for specific problems there to help me explore further. Often other researchers would approach me with more concrete problems, which is usually a very enlightening opportunity to learn about a new subarea and find more connections. I am a problem solver, and ultimately it is the concrete problems that keep me awake at night.

Mansour: When you are working on a problem, do you feel that something is true even before you have the proof?

Panova: Usually yes, but I could be dialectic. I would try to prove and disprove at the same time until it is clear which way it goes.

Mansour: What three results do you consider the most influential in combinatorics during the last thirty years?

Panova: Answering the question literally, influential would be the results which lead to a lot of subsequent work, and as such perhaps not specific Theorems, but rather conjectures or newly defined objects would top the list. Specifically, these would be (in no particular order): the discovery of cluster algebras by Fomin and Zelevinsky ${ }^{11}$; the study of dimer models starting with the work on the Aztec diamond initiated by Cohn-ElkiesKuperberg-Larsen-Propp ${ }^{12}$ and greatly expanded by Kenyon-Okounkov-Sheffield ${ }^{13}$ and evolved in the area of integrable probability driven by A. Borodin, I. Corwin, and many other famous mathematicians ${ }^{14}$. The study of diagonal harmonics with the flagship results the $n$ ! conjecture, proven by Haiman, and the subsequent "Shuffle conjecture" of HaglundHaiman-Loehr-Remmel-Ulyanov ${ }^{15}$, more re-

\footnotetext{
${ }^{6}$ G. Panova, Bijective enumeration of permutations starting with a longest increasing subsequence, Discrete Math. Theor. Comp. Sci. Proc. (2010), 973-982

${ }^{7}$ A. Crites, G. Panova, and G. Warrington, Separable permutations and Greene's theorem (2010), Ars Combin. 128 (2016), $103-116$

${ }^{8}$ G. Panova, Tableaux and plane partitions of truncated shapes (2010), Adv. in Appl. Math. 49:3-5 (2012), 196-217.

${ }^{9}$ Subsequently they independently solved and published this: R. Adin, R. King, Y. Roichman, Enumeration of standard Young tableaux of certain truncated shapes, The Electronic J. Combin. 18(2) (2011), \#P20.

${ }^{10}$ Integrable probability is the area at the intersection of statistical mechanics and probability which studies integrable particle models, see e.g. https://en.wikipedia.org/wiki/Integrable_system.

${ }^{11} \mathrm{~S}$. Fomin and A. Zelevinsky, Cluster algebras I: Foundations, J. Amer. Math. Soc. 15 (2002), $497-529$.

${ }^{12}$ N. Elkies, G. Kuperberg, M. Larsen, and J. Propp, Alternating-sign matrices and domino tilings, J. Algebraic Combin. $1: 111-132$ (1992) 219-234.

${ }^{13}$ R. Kenyon, A., Okounkov, and S. Sheffield, Dimers and amoebae, Ann. of Math. (2) 163 (2006), $1019-1056$.

${ }^{14}$ See https://www. claymath.org/events/cmi-himr-integrable-probability-summer-school.

${ }^{15}$ J. Haglund, M. Haiman, N. Loehr, J. B. Remmel, and A. Ulyanov, A combinatorial formula for the character of the diagonal coinvariants, Duke Math. J. 126 (2005), 195-232.
} 
cently proven by Carlsson-Mellit ${ }^{16}$, and all the work in symmetric functions these conjectures/results have motivated over the past 30 years. This is only within algebraic combinatorics, as of course, there have been major breakthrough results in other areas of combinatorics.

Mansour: What are the top three open questions in your list?

Panova: There is one major open problem - distinguishing the VP from VNP in arithmetic complexity theory ${ }^{17}$, which seems more accessible than $\mathrm{P}$ vs $\mathrm{NP}^{18}$. Specifically in algebraic combinatorics: the "combinatorial interpretation" of Kronecker coefficients and all the other "mysteriously" nonnegative coefficients and structure constants. Such interpretations may probably not exist, following Igor Pak's idea that Kronecker coefficients could be provably not in \#P (under some reasonable computational hypothesis).

Mansour: What kind of mathematics would you like to see in the next ten-to-twenty years as the continuation of your work?

Panova: I would like to see further development in the connections between algebraic combinatorics and computer science and probability. I hope to see progress towards the questions listed above.

Mansour: Do you think that there are core or mainstream areas in mathematics? Are some topics more important than others?

Panova: Yes. Most of the perceptions of "core" and "important" stem from the opinions of the experts and are often function on the number of researchers working in a given area. Yet there are many subareas with a lot of activity due largely to the variety and accessibility of the questions. I would measure the importance of an area by its connectivity to other areas of mathematics or other sciences and define its influences via the power and applicability of its methods. Then we would dis- tinguish the core.

Mansour: What do you think about the distinction between pure and applied mathematics that some people focus on? Is it meaningful at all in your case? How do you see the relationship between so-called "pure" and "applied" mathematics?

Panova: As long as theorems are being proven and mathematics is being developed, it could be considered "pure". I would consider "applied" that part of math that usually falls into modeling and engineering and solves their problems rather than proving new math theorems. Most of the mathematics we do and publish in journals like ECA qualifies as "pure" by that measure, and further division is meaningless.

Mansour: What advice would you give to young people thinking about pursuing a research career in mathematics?

Panova: Do mathematics for its beauty and the fun of the Eureka moment when you solve a problem. Do not be afraid of the long academic path because at every step there are multiple opportunities - math is used in so many areas in the industry besides academia, it is worth the journey.

Mansour: Would you tell us about your interests besides mathematics?

Panova: I have an interest in molecular biology and do some collaborations helping to model molecular dynamics in the DNA repair processes. While this is still "research work", it is unrelated to my main mathematical research career.

On a more personal level, I really like exploring nature. I go hiking, mountaineering, and I like to capture the beauty of nature with photographs. I used to paint and draw, but I have not pursued this much recently.

Mansour: In a series of four papers, coauthored with Morales and Pak ${ }^{19,20,21,22}$, on Hook formulas for skew shapes, you obtained

\footnotetext{
${ }^{16}$ E. Carlsson and A. Mellit, A proof of the shuffle conjecture, J. Amer. Math. Soc. 31 (2018), 661-697.

${ }^{17}$ For example, see A. Shpilka and A. Yehudayoff, Arithmetic Circuits: A Survey of Recent Results and Open Questions, Foundations and Trends in Theoretical Computer Science 5:3-4 (2010), 207-388.

${ }^{18}$ For example, see S. Aaronson, $P \stackrel{?}{=} N P$, in Open problems in mathematics, Springer, Cham, 2016, 1-122

${ }^{19}$ A. H. Morales, I. Pak, and G. Panova, Hook formulas for skew shapes I. q-analogues and bijections, J. Combin. Theory, Ser. A 154 (2018), 350-405.

${ }^{20}$ A. H. Morales, I. Pak, and G. Panova, Hook formulas for skew shapes II. Combinatorial proofs and enumerative applications, SIAM J. Discrete Math. 31 (2017), 1953-1989.

${ }^{21}$ A. H. Morales, I. Pak, and G. Panova, Hook formulas for skew shapes III. Multivariate and product formulas, Algebraic Combinatorics 2:5 (2019), 815-861.

${ }^{22}$ A. H. Morales, I. Pak, and G. Panova, Asymptotics for the number of standard Young tableaux of skew shape, European J. Combin. 70 (2018), 26-49.
} 
interesting and important results regarding the Naruse hook-length formula ${ }^{23}$ which has already motivated a great deal of recent interest in the enumeration of skew tableaux and related topics. Would you tell us about this formula, the main motivation behind this project, and some potential applications of techniques developed in these papers? Will this series continue with new surprising results and applications?

Panova: One of the most fascinating classical results in algebraic combinatorics is the Hooklength formula of Frame-Robinson-Thrall ${ }^{24}$ for the number $f^{\lambda}$ of Standard Young Tableaux of a given shape $\lambda$, equivalently the dimension of the irreducible $S_{n}$ representation of the Specht module of $\lambda$, which gives $f^{\lambda}$ as $n$ ! divided by the product of hooks of $\lambda$. For SYTs of skew shapes no such formula exists, as can be seen from the special case of $f^{\delta_{n} / \delta_{n-2}}$ given by the Euler numbers. In $2014 \mathrm{Hi}$ roshi Naruse presented a remarkable formula expressing $f^{\lambda / \mu}$ as $n$ ! times a sum over "excited" diagrams of $\mu$ inside $\lambda$ weighted by the inverse product of hook lengths of each diagram. Naturally, such a beautiful result needed a "combinatorial" explanation and we set on to [re]prove it by first generalizing it to $q$-weighted enumeration over skew SSYTs and reverse plane partitions and proving those results algebraically, bijectively, and later on via recursions and non-intersecting lattice paths. Thanks to the positive-sum and nice weights the formula proved to be useful for applications, mostly related to asymptotic results (for the number of skew SYTs to lozenge tilings and more refined asymptotics and probabilistic behavior of SYTs). I am expecting to see more such asymptotic applications of this formula and I am hoping for further connections to Schur functions. The new, though not entirely surprising, results concern its deformations, for example to Grothendieck polynomials and the associated standard increasing tableaux.

Mansour: Kronecker coefficients, as 'tools' from Representation theory, are used to describe the decomposition of the tensor product of two irreducible representations of a symmetric group into irreducible representations. How do they come into play in combinatorics in general and in your research in particular?

Panova: Ever since their introduction by Murnaghan $^{25}$ more than 80 years ago, they have posed a major problem - to give any kind of positive combinatorial formula for them. Kronecker coefficients can be expressed using characters of $S_{n}$, Schur functions, and even contingency arrays. Yet, their inherent nonnegativity (as multiplicities of irreducible representations) and their semigroup property are completely mysterious from all these (nonpositive) combinatorial formulas. Ever since we started looking at them with Igor Pak, I have been trying to understand them and answer this question, perhaps in the negative formally using computational complexity notions. The various formulas have given us a lot of partial results, and also implications to other problems (like Sylvester's ${ }^{26}$ unimodality for the number of integer partitions inside a rectangle, ref. our papers with Igor Pak). A major motivation to study them came from GCT, where they can be used to understand the representation theory of the coordinate rings for the determinant and permanent and thus hopefully distinguish them and possibly prove that $V P \neq V N P$. In the course of our investigations, we disproved a cornerstone conjecture in $\mathrm{GCT}^{5}$, but we also understood more about them.

Mansour: In the paper entitled Asymptotics of symmetric polynomials with applications to statistical mechanics and representation theory, co-authored by Gorin ${ }^{27}$, you developed a new method for studying the asymptotics of symmetric polynomials of representationtheoretic origin as the number of variables tends to infinity. Would you explain this method and some of its applications briefly? Models from statistical physics have always

\footnotetext{
${ }^{23}$ H. Naruse, Schubert calculus and hook formula, talk slides at 73rd Sém. Lothar. Combin., Strobl, Austria, 2014; available at tinyurl.com/z6paqzu.

${ }^{24}$ J. S. Frame, G. de B. Robinson, and R. M. Thrall, The hook graphs of the symmetric group, Canad. J. Math. 6 (1954), $316-324$.

${ }^{25}$ D. Murnaghan, The analysis of the direct product of irreducible representations of the symmetric groups, Amer. J. Math. 60:9 (1938), 44-65.

${ }^{26}$ J. J. Sylvester, Proof of the hitherto undemonstrated fundamental theorem of invariants, Philos. Mag. 5 (1878), 178-188, reprinted in: Coll. Math. Papers, vol. 3, Chelsea, New York, 1973, 117-126.

${ }^{27} \mathrm{~V}$. Gorin and G. Panova, Asymptotics of symmetric polynomials with applications to statistical mechanics and representation theory, Ann. Probab. 43:6 (2015), 3052-3132.
} 
been a rich source of interesting problems for combinatorics and probability. Are there specific problems in this direction in your research plan for the near future?

Panova: Indeed, as I have mentioned above, integrable probability uses symmetric function theory. The normalized Schur functions can be interpreted as a variant of the moment generating functions for observables in the lozenge tiling model (e.g. positions of horizontal lozenges near the boundary). Their asymptotic behavior gives us the probability distributions of certain lozenges and can be further used to derive limit surfaces and behavior of such dimer models. These methods, however, rely strongly on the geometry of the domain/boundary conditions and dimer weights, and extending our understanding further is still ongoing. Some specific problems concern the asymptotics of normalized skew Schur functions or the study of tiling models with long-range interaction which we encountered when studying the seemingly unrelated problem on maximal Schubert polynomials.

Mansour: Combinatorics is full of important formulas. Which three are your favorite?

Panova: The original hook-length formula for the number of $\mathrm{SYTs}^{23}$ has been a major source of inspiration and beautiful results. The Lindström $^{28}$, Gessel-Viennot ${ }^{29}$, and KarlinMacGregor ${ }^{30}$ determinantal formula for counting non-intersecting lattice paths has been useful across the areas from algebra to probability. The matrix-tree theorem and Weyl determinantal formula are also up on the list.

Mansour: You were one of the panelists at the workshop, "Towards a new theoretical biology," in 2018 at the University of Pennsylvania. Would you tell us about the main differences between the new and the old? How does combinatorics involve there?

Panova: I believe I was on this panel because I am a mathematician with some work in molecular biology, but it is unrelated to my work in combinatorics. The discussion in this workshop was focused on why biology has not been able to take such advantage of mathematical methods and developed as theoretical physics has. The "old" theoretical biology revolved mostly around models in population ge- netics and reaction kinetics. Subsequently, the fields of systems biology and computational biology (genetics and neuroscience) emerged, but their level of abstraction and generality is nowhere close to what we have seen in theoretical physics. The main issue comes from the inherent complexity of living systems and life's uniqueness. Despite the great variety of living organisms, the underlying biology is all too similar in its building blocks and basic mechanism and at the same, these are too complex to pick apart.

Mansour: You have extensive experience with major mathematics competitions such as International Mathematics Olympiads and Putnam. Do you think that mathematics competitions play a crucial role to inspire young students for a research career?

Panova: Mathematical competitions definitely help popularize mathematics among students and also help them develop problemsolving skills, which are useful also outside academic math like tech and finance jobs. The caveat is that this very inspiring (and fun!) experience could also be slightly misleading since, unlike competitions, research problems are rarely solvable in 3 hours.

Mansour: In your work, you have extensively used combinatorial reasoning to address important problems. How do enumerative techniques engage in your research?

Panova: Enumerative techniques help from the beginning by breaking the problem into possibly smaller structures, making simpler models, finding symmetries and recursions. Generating functions are also a very powerful tool by itself.

Mansour: It is a fact that not many women follow a professional career in mathematics. It is a discussion around the globe on how to get more women into mathematics. What do you think about this issue? What should be done in the next ten years to involve more women in mathematics?

Panova: Algebraic combinatorics is doing something right, as it seems that the proportions of women in this field are higher than in most other areas of math. A lot of the current efforts focus on recruitment at later stages, but I think the issues start already in

\footnotetext{
${ }^{28} \mathrm{~B}$. Lindström, On the vector representation of induced matroids, Bull. London Moth. Sot. 5 (1973), 85-90.

${ }^{29}$ I. Gessel and G. Viennot, Binomial determinants, paths, and Hook length formulae, Adv. Math.58 (1985), 300-321.

${ }^{30}$ S. Karlin and J. G. McGregor, Coincidence probabilities, Pacific J. Math. 9 (1959), 1141-1164.
} 
middle school. There are too many stereotypes poisoning the experience from early on - from gender biases to the unpopularity of math as a subject (especially in the US). We should each do our part in debunking these stereotypes at every level.

Mansour: You are the recipient of the IMI Prize for the year 2020, a prize awarded by the Institute of Mathematics and Informatics of the Bulgarian Academy of Sciences. The prize will be presented during the International Conference "Mathematics Days of in Sofia" in 2021. How do you feel about going back to the city you were born to obtain a prize in mathematics? Do you think that you will be whispering, "yes, I did it, grandma!" while your eyes will have been filling with tears?

Panova: Too bad my grandmas died more than 20 years ago... It is a great honor to be recognized in my own land ${ }^{31}$ and it is especially important to me as an opportunity to introduce my field of algebraic combinatorics which is not represented in Bulgaria.

Mansour: You have some of your paintings on your web page. Have you ever planned to be a painter? How would you compare the two creative activities: painting and doing mathematics? Stendhal syndrome, a strange aesthetic sickness, is described as a psychosomatic condition involving rapid heartbeat and fainting occurring when an individual becomes exposed to artworks of great beauty. Do you think that we, mathematicians, experience a similar syndrome when we see an outstanding result or an excellent paper? If yes, would you like to offer a name to this syndrome?

Panova: Up until college I was planning to be an architect which seemed a good combination of math and painting. Aesthetics in art and math could be indeed quite similar to experiences. But unlike art, which can be widely appreciated, recognizing mathematical beauty requires more expertise. Personally, my enjoyment of both math and art is in practicing them - the joy and satisfaction for finding a nice solution are quite similar to those I had experienced by expressing on paper the image in my head.

Mansour: Would you tell us about your thought process for the proof of one of your favorite results? How did you become interested in that problem? How long did it take you to figure out the proof? Did you have a "eureka moment"?

Panova: I must say that the older and more experienced I get, the less surprise and excitement of the "eureka moment" I have. Recently my solutions have been gradually built up from smaller problems and often result from finding connections. My favorite results are "Rectangular Kronecker coefficients and plethysms in geometric complexity theory" 4 (with C. Ikenmeyer), "Asymptotics of symmetric polynomials with applications to statistical mechanics and representation theory" 26 (with V. Gorin), and "Hook-length formulas for skew shapes I" 18 (with A. Morales and I. Pak). These were all multilayered projects and no problem there was solved at once. For the sake of example, without being too concrete, I can describe a typical process: first I would try to understand the objects in the problem usually by interpreting them geometrically or algebraically (e.g. using symmetric functions), and I try to visualize the problem as much as possible. Unlike many combinatorialists, I rarely start with doing small examples, I would rather encode the structure in full generality, look for invariants, special characteristics, etc. Exploring different models ultimately could lead to a more "familiar" situation that can be handled with existing tools.

Mansour: Is there a specific problem you have been working on for many years? What progress have you made?

Panova: Kronecker coefficients have always been burning a hole in my mind. We have certainly understood them better throughout the past 10 years, and that research has led to many other interesting connections and developments. At the same time, the problem on whether they are in \#P or not is still out there with no solution in sight, so it would be hard to tell how close we are.

Mansour: Professor Greta Panova, I would like to thank you for this very interesting interview on behalf of the journal Enumerative Combinatorics and Applications.

${ }^{31}$ but I can't be a prophet there per the old saying. 